\title{
Evolução hemodinâmica da revascularização do miocárdio com dois métodos de proteção miocárdica
}

\author{
Paulo M. PÊGO-FERNANDES*, Fabio B. JATENE*, Fabricio Ferreira COELHO**, \\ André Felix GENTIL**, Karina L. KAWASNICKA*, Noedir A. G. STOLF*, Sérgio A. OLIVEIRA*
}

RBCCV 44205-504

Pêgo-Fernandes P M, Jatene F B, Coelho F F, Gentil A F, Kawasnicka K L, Stolf N A G, Oliveira S A - Evolução hemodinâmica da revascularização do miocárdio com dois métodos de proteção miocárdica. Rev Bras Cir Cardiovasc 2000; 15(3): 212-8.

RESUMO: Objetivo: Avaliar a evolução hemodinâmica imediata na revascularização do miocárdio com pinçamento intermitente da aorta, acrescido ou não de um protocolo de pré-condicionamento.

Casuística e Métodos: Trinta e cinco pacientes submetidos à revascularização do miocárdio foram randomizados em 2 grupos. No grupo controle (18), o procedimento foi realizado com pinçamento intermitente da aorta; no segundo grupo (17), denominado pré-condicionamento, foram acrescidos 2 pinçamentos curtos da aorta, previamente ao pinçamento intermitente convencional. Foram obtidos a pressão arterial média (PAm), a pressão capilar pulmonar (PCP), o índice cardíaco (IC) e o índice de trabalho sistólico do VE (ITSVE) antes da circulação extracorpórea (1); antes da sutura esternal (2); com 6h (3); 12h (4); 18h (5) e 24h de pósoperatório (6), além da fração de ejeção (FEVE) por ecocardiograma nos momentos 1 e 2.

Resultados: Não houve diferença estatística entre os grupos, havendo um aumento do IC e FEVE após a revascularização. Todos os pacientes tiveram boa evolução clínica.

Conclusão: O comportamento hemodinâmico foi semelhante nos dois grupos de pacientes.

DESCRITORES: Revascularização miocárdica. Revascularização miocárdica, métodos. Hemodinâmica, fisiologia. Circulação extracorpórea. Aorta, pinçamento. Miocárdio, proteção. Revascularização miocárdica, estudo comparativo. Revascularização miocárdica, hemodinâmica.

\section{INTRODUÇÃO}

Um dos questionamentos em relação à técnica de pinçamento intermitente da aorta é de que a repetição de episódios de isquemia, que individualmente seriam reversíveis, poderiam causar danos cumulativos com conseqüente necrose. Esta afirmativa foi negada por REIMER et al. (1), que mostraram experimentalmente que a reperfusão intermitente previne o déficit metabólico cumulativo e a isquemia miocárdica com morte celular, e que um primeiro episódio de isquemia reduz o consumo dos fosfatos de alta energia nos episódios subseqüentes. Outros estudos (2) mostraram que breves períodos de isquemia não induzem dano celular irreversível e que eles não são cumulativos nos déficits metabólicos, estruturais e funcionais. Foi ainda documentado que breves períodos de isquemia aumentam a tolerância do coração ao invés de torná-lo mais

Trabalho realizado no Instituto do Coração do Hospital das Clínicas da Faculdade de Medicina da Universidade de São Paulo. São Paulo, SP, Brasil. Recebido para publicação em fevereiro de 2000.

* Da Divisão de Cirurgia do Instituto do Coração do Hospital das Clínicas da Faculdade de Medicina da Universidade de São Paulo.

** Da Faculdade de Medicina da Universidade de São Paulo.

Endereço para correspondência: Paulo M. Pêgo-Fernandes. Av. Dr. Enéas de Carvalho Aguiar, $44-2^{\circ}$ andar. São Paulo, SP, Brasil. CEP $05403-000$.

Fone/Fax: (11) 3069-5248. e-mail: paulopego@ incor.usp.br 
Pêgo-Fernandes P M, Jatene F B, Coelho F F, Gentil A F, Kawasnicka K L, Stolf N A G, Oliveira S A - Evolução hemodinâmica da revascularização do miocárdio com dois métodos de proteção miocárdica. Rev Bras Cir Cardiovasc 2000; 15(3): 212-8.

vulnerável aos episódios subseqüentes de isquemia. Essa indução da tolerância à isquemia foi denominada de pré-condicionamento isquêmico (3).

O pré-condicionamento isquêmico foi descrito por MURRY et al. (3), que demonstraram em experimentos com animais que, no coração submetido à isquemia reversível intermitentemente com períodos de reperfusão, o miocárdio torna-se resistente ao infarto após um tempo isquêmico prolongado, o que anteriormente levaria a um dano irreversível.

Vários estudos indicam que o pré-condicionamento isquêmico limita o tamanho do infarto, melhorando a função ventricular pós-isquêmica e parâmetros hemodinâmicos, quando associado a outros métodos de proteção miocárdica (4-6).

O objetivo deste trabalho é avaliar a evolução hemodinâmica imediata, na revascularização do miocárdio com pinçamento intermitente da aorta, acrescido ou não de um protocolo de pré-condicionamento.

\section{CASUÍSTICA E MÉTODOS}

No período de outubro de 1998 a maio de 1999, 35 pacientes portadores de insuficiência coronária com indicação de operação de revascularização do miocárdio foram divididos de maneira prospectiva e randomizada em 2 grupos. O primeiro grupo, denominado de controle, foi operado da forma habitualmente utilizada pelos dois cirurgiões envolvidos: hipotermia sistêmica a $32^{\circ} \mathrm{C}$ com pinçamento intermitente da aorta e uso de circulação extracorpórea. No segundo grupo, denominado de pré-condicionamento, foram acrescidos dois pinçamentos curtos da aorta com intervalo de reperfusão entre eles, previamente ao pinçamento intermitente da forma convencional.

Dezoito pacientes ficaram no grupo controle e 17 no grupo pré-condicionamento. Os critérios de inclusão foram: fração de ejeção pré-operatória maior que $30 \%$, não ser reoperação, ter no mínimo lesão de 2 artérias coronárias, estar previsto o uso de circulação extracorpórea, não apresentar, no momento da operação, angina instável, não estar na fase aguda do infarto do miocárdio, não necessitar de outras correções associadas.

Em todos os pacientes utilizou-se a canulação da aorta ascendente e das duas veias cavas para a instalação da circulação extracorpórea. Era, então, introduzido um cateter da marca $3 \mathrm{M}$, habitualmente utilizado para retroplegia, no seio coronário, e, através dele, eram feitas as coletas sangüíneas do mesmo. Somente neste momento o cirurgião e demais membros da equipe tomavam conhecimento se o paciente pertencia ao grupo controle ou précondicionamento.

Após o resfriamento sistêmico para $32^{\circ} \mathrm{C}$, eram iniciados os pinçamentos da aorta. No grupo controle, durante cada pinçamento da aorta, era realizada uma anastomose entre o enxerto e a artéria coronária a ser revascularizada. Para cada 3-4 min de pinçamento, realizamos $1 \mathrm{~min}$ de reperfusão. Esse procedimento foi utilizado tanto para as anastomoses distais quanto para as proximais. No grupo de précondicionamento isquêmico, eram realizados dois pinçamentos da aorta de $3 \mathrm{~min}$ de duração, entremeados por 2 min de reperfusão. Após o segundo intervalo de reperfusão, a operação era realizada de forma idêntica ao grupo controle. Durante a realização da última anastomose, era iniciado o aquecimento sistêmico do paciente.

As medidas hemodinâmicas das pressões capilar pulmonar (PMCP), e arterial sistêmica (PAM), em $\mathrm{mmHg}$, e a aferição do débito cardíaco (DC) foram registradas através da utilização do apareIho da marca Bese, modelo Biomonitor 07. O valor do $\mathrm{DC}$, calculado em $\mathrm{l} / \mathrm{min}$, foi obtido pela média aritmética de três determinações. Todas essas medidas e registros eram feitos nos seguintes momentos: 1- antes da circulação extracorpórea; 2 antes de iniciar a sutura esternal; 3- com $6 \mathrm{~h}$ de pós-operatório; 4- com 12 h de pós-operatório; 5com 18 h de pós-operatório; 6- com 24 h de pósoperatório.

Os parâmetros hemodinâmicos derivados foram calculados a partir de fórmulas clássicas, pelo próprio aparelho: 1- Índice cardíaco (IC); 2- Índice de trabalho sistólico do ventrículo esquerdo (ITSVE).

Foram realizadas medidas da fração de ejeção através do uso de ecocardiografia transesofágica, sendo estas feitas antes da circulação extracorpórea e da sutura esternal. O aparelho de ecocardiograma utilizado foi o Apogee CX, marca ATL.

\section{Análise Estatística}

Com relação à análise estatística, o Teste Exato de Fisher foi empregado para avaliar comparativamente os dois grupos quanto à homogeneidade de proporções. Os testes t-Student e de Wilcoxon foram empregados para avaliar comparativamente os dois grupos quanto aos dados quantitativos.

Para as variáveis de hemodinâmica, procurouse estudar o comportamento ao longo dos momentos nos dois grupos através da Análise de Variância para Medidas Repetidas. Quando necessário, considerou-se matrizes de covariâncias diferentes para os dois grupos. O nível de significância estabelecido para análise foi de $5 \%$ e todos os cálculos foram 
Pêgo-Fernandes P M, Jatene F B, Coelho F F, Gentil A F, Kawasnicka K L, Stolf N A G, Oliveira S A - Evolução hemodinâmica da revascularização do miocárdio com dois métodos de proteção miocárdica. Rev Bras Cir Cardiovasc 2000; 15(3): 212-8.

realizados por meio do sistema SAS (Statistical Analysis System).

Todas as compilações de dados hemodinâmicos e a análise estatística foram realizadas por pessoas que desconheciam a qual grupo o paciente pertencia.

\section{RESULTADOS}

Não houve diferenças entre os dois grupos de pacientes em relação às características pré-operatórias, tais como idade, sexo, peso, altura, incidência de diabete melito, presença de infarto do miocárdio prévio, presença de bloqueio de ramo ao eletrocardiograma, angina instável prévia, confirmando a homogeneidade entre os grupos.

Em relação às características intra-operatórias, incluindo tempo máximo de pinçamento da aorta, soma do tempo de pinçamentos da aorta, número de artérias coronárias revascularizadas, tempo de circulação extracorpórea, tempo de anestesia e cirurgião envolvido, também não houve diferença entre os dois grupos de pacientes. Foram realizados em média 3,3 enxertos/paciente no grupo controle e 3,2 enxertos/paciente no grupo pré-condicionamento. Nenhum paciente apresentou sinais de infarto miocárdico intra-operatório.

Quanto aos parâmetros hemodinâmicos medidos ou calculados, nos diversos momentos, notamos os seguintes resultados: apenas nos pacientes pertencentes ao grupo controle, a pressão arterial média apresentou elevação significativa no momento $6 \mathrm{em}$ relação ao momento 1 (Gráfico 1). A pressão capilar pulmonar não apresentou diferença nem em relação aos momentos, tampouco em relação aos grupos de pacientes.

O índice cardíaco mostrou comportamento semelhante nos dois grupos, não havendo diferença estatisticamente significante. Entretanto, houve variação do índice cardíaco ao longo do tempo (Gráfico 2). Constatou-se que houve aumento significativo desse índice no momento 2 (antes da sutura esternal), em relação aos demais momentos $(p<0,001)$. O índice de trabalho sistólico do ventrículo esquerdo não apresentou diferença nem em relação aos momentos, tampouco em relação aos grupos de pacientes.

Em relação à fração de ejeção, houve um comportamento semelhante ao longo do tempo nos dois grupos, não havendo diferença estatisticamente significante entre os perfis médios dos dois grupos. Em ambos os grupos constatou-se um aumento significativo da fração de ejeção antes da sutura esternal $(p=0,006)$ - Gráfico 3. Os dados completos dessas variáveis estão na Tabela 1 .

\section{GRÁFICO 1}

REPRESENTAÇÃO GRÁFICA DA EVOLUÇÃO DOS VALORES DA PRESSÃO ARTERIAL (PA), EM MÉDIA, AO LONGO DOS TRÊS MOMENTOS DE DOSAGEM

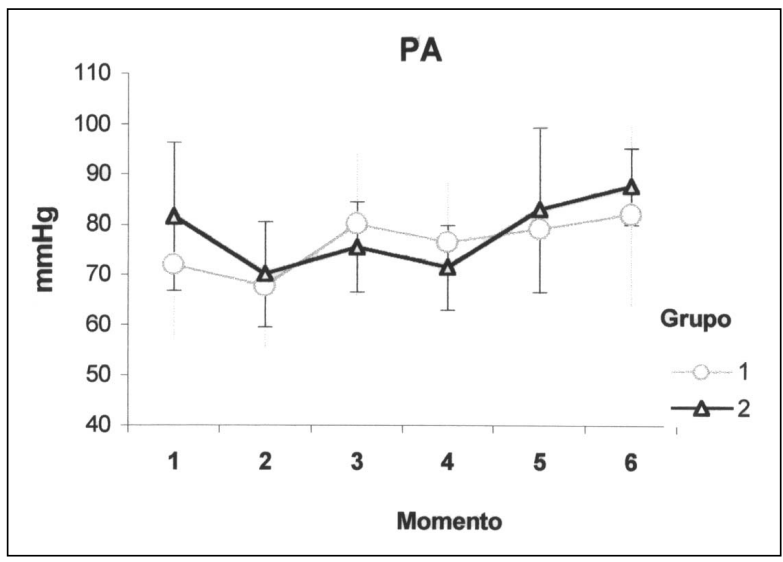

\section{GRÁFICO 2}

REPRESENTAÇÃO GRÁFICA DA EVOLUÇÃO DOS

VALORES DE ÍNDICE CARDÍACO (IC), EM MÉDIA, AO LONGO DOS TRÊS MOMENTOS DE DOSAGEM

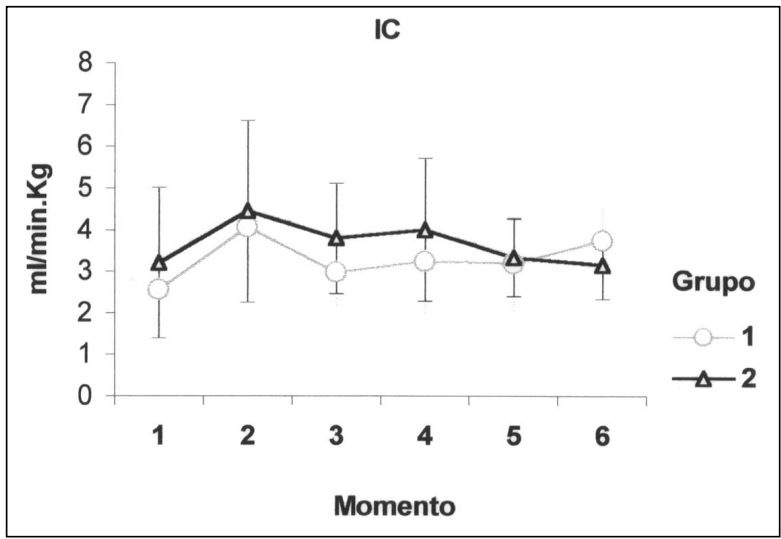

GRÁFICO 3

REPRESENTAÇÃO GRÁFICA DA EVOLUÇÃO DOS VALORES DE FRAÇÃO DE EJEÇÃO DO VENTTRÍCULO ESQUERDO (FE), EM MÉDIA, AO LONGO DOS DOIS MOMENTOS DE AVALIAÇÃO

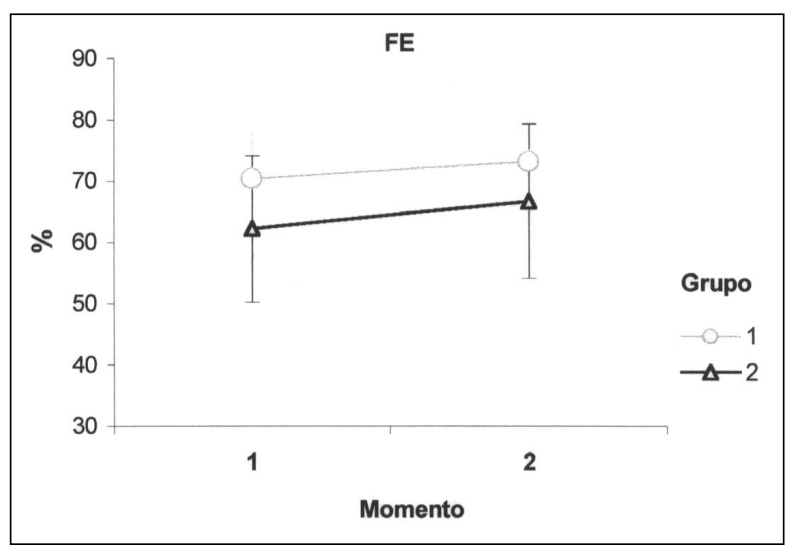


Pêgo-Fernandes P M, Jatene F B, Coelho F F, Gentil A F, Kawasnicka K L, Stolf N A G, Oliveira S A - Evolução hemodinâmica da revascularização do miocárdio com dois métodos de proteção miocárdica. Rev Bras Cir Cardiovasc 2000; 15(3): $212-8$.

TABELA 1

\begin{tabular}{|c|c|c|c|c|c|c|}
\hline \multicolumn{7}{|c|}{$\begin{array}{l}\text { COMPORTAMENTO DE DIVERSOS PARÂMETROS NO GRUPO CONTROLE E } \\
\text { PRÉ-CONDICIONAMENTO DE } 6 \text { MOMENTOS ESTUDADOS }\end{array}$} \\
\hline & $\begin{array}{l}\text { MOMENTO1 } \\
\text { CONTR. PRÉ }\end{array}$ & $\begin{array}{l}\text { MOMENTO2 } \\
\text { CONTR. PRÉ }\end{array}$ & $\begin{array}{l}\text { MOMENTO3 } \\
\text { CONTR. PRÉ }\end{array}$ & $\begin{array}{l}\text { MOMENTO4 } \\
\text { CONTR. PRÉ }\end{array}$ & $\begin{array}{l}\text { MOMENTO5 } \\
\text { CONTR. PRÉ }\end{array}$ & $\begin{array}{l}\text { MOMENTO6 } \\
\text { CONTR. PRÉ }\end{array}$ \\
\hline PAm & $\begin{array}{l}72,17 \pm 15,00 \\
76,61 \pm 12,16\end{array}$ & $\begin{array}{l}81,59 \pm 14,52 \\
71,50 \pm 8,46\end{array}$ & $\begin{array}{l}67,88 \pm 12,42 \\
79,24 \pm 11,21\end{array}$ & $\begin{array}{l}70,12 \pm 10,55 \\
82,93 \pm 16,28\end{array}$ & $\begin{array}{l}80,22 \pm 14,22 \\
82,13 \pm 18,01\end{array}$ & $\begin{array}{l}75,56 \pm 8,94 \\
87,67 \pm 7,62\end{array}$ \\
\hline IC & $\begin{array}{l}2,56 \pm 0,74 \\
3,24 \pm 1,22\end{array}$ & $\begin{array}{l}3,20 \pm 1,81 \\
4,00 \pm 1,72\end{array}$ & $\begin{array}{l}4,07 \pm 1,17 \\
3,20 \pm 1,14\end{array}$ & $\begin{array}{l}4,44 \pm 2,18 \\
3,33 \pm 0,93\end{array}$ & $\begin{array}{ll}2,99 \pm & 0,87 \\
3,75 \pm & 0,83\end{array}$ & $\begin{array}{l}3,79 \pm 1,32 \\
3,14 \pm 0,80\end{array}$ \\
\hline PCP & $\begin{array}{r}9,72 \pm \quad 4,72 \\
11,22 \pm 4,19\end{array}$ & $\begin{array}{l}11,41 \pm 4,73 \\
11,20 \pm 4,89\end{array}$ & $\begin{array}{l}11,22 \pm \quad 4,94 \\
11,12 \pm 5,89\end{array}$ & $\begin{array}{l}12,35 \pm 5,42 \\
12,87 \pm 7,29\end{array}$ & $\begin{array}{l}10,11 \pm 6,70 \\
13,50 \pm 15,35\end{array}$ & $\begin{array}{l}14,06 \pm 5,93 \\
13,27 \pm 10,68\end{array}$ \\
\hline ITSVE & $\begin{array}{l}38,89 \pm 17,12 \\
34,63 \pm 12,59\end{array}$ & $\begin{array}{l}44,51 \pm 19,11 \\
40,32 \pm 18,08\end{array}$ & $\begin{array}{l}42,18 \pm 14,75 \\
35,42 \pm 12,84\end{array}$ & $\begin{array}{l}37,39 \pm 19,29 \\
41,89 \pm 18,81\end{array}$ & $\begin{array}{l}32,29 \pm 10,92 \\
44,02 \pm 19,70\end{array}$ & $\begin{array}{l}36,89 \pm 10,03 \\
39,53 \pm 10,02\end{array}$ \\
\hline FEVE & $70,40 \pm 7,78$ & $62,17 \pm 11,99$ & $73,22 \pm 6,34$ & $86,67 \pm 12,55$ & & \\
\hline
\end{tabular}

PAm: momento $1 \times$ momento 6 no grupo controle $(p=0,44)$

IC: momento 1 e 3-6 $x$ momento 2 em ambos os grupos $(p<0,001)$

FEVE: momento $1 \times$ momento 2 em ambos os grupos $(p=0,006)$

\section{COMENTÁRIOS}

O pré-condicionamento isquêmico miocárdico pode ser definido como um mecanismo protetor endógeno, no qual curtos períodos de isquemia e reperfusão conferem ao miocárdio resistência a um insulto isquêmico mais prolongado ${ }^{(5,7)}$. Desde sua descrição inicial por MURRY et al. (3), em 1986, por meio de um modelo canino, este mecanismo adaptativo foi demonstrado em todas as espécies animais estudadas ${ }^{(8)}$, com alguns autores sugerindo sua existência no homem ${ }^{(9,10)}$.

No coração, além de atuar sobre o desenvolvimento do infarto, o pré-condicionamento isquêmico também reduz as arritmias induzidas pelo mecanismo isquemia-reperfusão ${ }^{(11)}$, o que pode melhorar a evolução hemodinâmica pós-operatória dos pacientes submetidos a esta técnica.

A função ventricular é o principal determinante do risco cirúrgico e sobrevida tardia dos pacientes submetidos à revascularização do miocárdio (12). O baixo débito cardíaco no pós-operatório, secundário à disfunção ventricular, ainda é um problema que aumenta os custos e morbimortalidade em cirurgia cardíaca, sendo uma das principais causas de óbito no pós-operatório imediato ${ }^{(6)}$.

Vários autores estudaram a função ventricular e evolução hemodinâmica imediata após o uso de protocolo de pré-condicionamento isquêmico. ILLES \& SWOYER ${ }^{(6)}$ estudaram a aplicabilidade do précondicionamento a pacientes submetidos à proteção miocárdica com o uso de solução cardioplégica sangüínea intermitente. Em um estudo prospectivo e randomizado, utilizaram um protocolo de pré-condi- cionamento, em que era realizado um pinçamento da aorta de $1 \mathrm{~min}$ em normotermia, seguido de 5 min de reperfusão. Notaram um aumento de CKMB ao longo do tempo, porém não significativamente diferente no grupo controle e no grupo pré-condicionado. Notaram, porém, uma grande melhora dos índices hemodinâmicos, principalmente do índice cardíaco dos valores pré-operatórios, ao contrário do grupo controle, que teve deterioração do índice só voltando ao nível basal após 6 a 12 horas, com utilização bem menor de drogas inotrópicas no grupo pré-condicionado. Em sua discussão, levantam a hipótese de que a grande melhora dos índices hemodinâmicos possa ser devida à menor quantidade de miocárdio "atordoado". Sugerem que esse miocárdio "atordoado" possa ser na verdade um pequeno grau de necrose celular não detectável pela CKMB e, possivelmente, detectável pela troponina. Essas duas afirmações não encontram respaldo na literatura. Em relação ao "atordoamento" miocárdico, estudos experimentais (13) mostraram que o pré-condicionamento não o reduziria. Neste mesmo trabalho(6), os autores não encontraram diferença significativa nos valores da pressão venosa central e na pressão capilar pulmonar no pré e pós-operatório entre o grupo controle e o submetido ao pré-condicionamento, o que corrobora nossos resultados. Entretanto, FLAMENG et al. (14) encontraram diferença na pressão capilar pulmonar no pré e pós-operatório de pacientes submetidos a pinçamento intermitente em hipotermia, porém não em normotermia.

Quanto à pressão arterial média, os trabalhos que avaliaram este parâmetro têm resultados vari- 
Pêgo-Fernandes P M, Jatene F B, Coelho F F, Gentil A F, Kawasnicka K L, Stolf N A G, Oliveira S A - Evolução hemodinâmica da revascularização do miocárdio com dois métodos de proteção miocárdica. Rev Bras Cir Cardiovasc 2000; 15(3): 212-8.

áveis quanto ao seu comportamento após o emprego de pré-condicionamento isquêmico. Em nosso estudo, encontramos no grupo controle um aumento significativo no momento 6 em relação ao momento 1, o que concorda com os resultados de FLAMENG et al. (14). Já outros autores encontraram diminuição significativa tanto no grupo controle quanto no précondicionado após $12 \mathrm{~h}$, explicando este fato por uma provável diminuição do tônus parassimpático (6).

Existem vários outros trabalhos na literatura comparando o uso do pré-condicionamento isquêmico juntamente com outros métodos de proteção miocárdica, apresentando estes resultados variáveis quanto ao incremento no grau de proteção e melhora da função hemodinâmica no pós-operatório. CREMER et al. ${ }^{(4)}$ conduziram um estudo prospectivo em que em um grupo realizou-se précondicionamento normotérmico, com 2 pinçamentos de 5 minutos antes do uso de cardioplegia gelada; no grupo controle apenas a cardioplegia foi utilizada. Como conclusão, os autores não encontraram diferença significante entre os grupos com relação aos parâmetros bioquímicos (lactato, CKMB e troponina T), porém a função cardíaca melhorou quando o pré-condicionamento foi aplicado. Outros autores (12) compararam o uso da cardioplegia quente com aspartato-glutamato e o pinçamento intermitente como métodos de proteção miocárdica, estudando vários parâmetros hemodinâmicos. Encontraram um aumento do índice cardíaco e índice de trabalho do VE em ambos os grupos, sem diferença significativa entre eles, concluindo-se, portanto, pela equivalência dos métodos quanto à sua eficácia na preservação funcional do miocárdio. Em nosso estudo, no entanto, não encontramos diferença no índice de trabalho sistólico do VE, nem em relação aos momentos tampouco em relação aos grupos de pacientes.

O estudo da utilização do pré-condicionamento isquêmico em pacientes submetidos à revascularização do miocárdio minimamente invasiva é uma situação especialmente interessante, já que os pacientes estão em normotermia, com o coração batendo, não descomprimido e exercendo o trabaIho ventricular necessário à manutenção das condições hemodinâmicas. Em um destes estudos(15) ocluiu-se a artéria interventricular anterior durante 5 min, com reperfusão também de $5 \mathrm{~min}$. Após este período, fez-se nova oclusão para a realização da anastomose entre a artéria torácica interna e a artéria coronária interventricular anterior. Estudando o comportamento do ventrículo esquerdo e da pressão da artéria pulmonar através do uso de ecocardiograma transesofágico, não se observou diferença de comportamento desses parâmetros entre a primeira e a segunda oclusão coronária. Concluiu-se que não houve um benefício desse primeiro período de isquemia.

TOMODA \& AOKI (16), por sua vez, acharam efeito protetor significativo com melhora da FEVE e diminuição da porcentagem de falência cardíaca pósisquêmica, nos pacientes com angina prévia submetidos a trombólise, mas não acharam diferença para os tratados com angioplastia transluminal. O estudo de pacientes submetidos a trombólise intracoronariana (17) encontrou melhora significativa da FEVE e motilidade miocárdica nos pacientes com angina até 4 horas antes do IAM, observando, ainda, que os pacientes com melhor circulação colateral na fase aguda tiveram melhor evolução após o procedimento, por uma provável potencialização do efeito benéfico do pré-condicionamento isquêmico. Outros $^{(18)}$ não encontraram diferença significativa na circulação colateral cardíaca nem na FEVE e motilidade da parede anterior em grupos controle e submetido a pré-condicionamento.

Em nosso estudo, verificamos o comportamento ecocardiográfico do ventrículo esquerdo antes e depois da circulação extracorpórea no intra-operatório; notamos aumento da fração de ejeção, compatível com o aumento do índice cardíaco encontrado nesse mesmo período. Acreditamos que a ação de drogas vasoativas, assim como de vasodilatadores, empregados de rotina em nosso Serviço, além da melhora da isquemia miocárdica, possam explicar esses resultados. Nestes parâmetros não encontramos diferença entre o grupo controle e pré-condicionado.

\section{CONCLUSÃO}

Concluímos que a evolução hemodinâmica imediata foi semelhante nos dois grupos, sendo os dois métodos igualmente eficientes. Apesar dos avanços em seu conhecimento e utilização, o estudo do pré-condicionamento isquêmico é ainda relativamente recente, e o seu real benefício ainda é uma questão em aberto.

AGRADECIMENTOS: Carina Savalli Redigolo (análise estatística), Tânia Polon de Mendonça, Dra. Célia M. C. Strunz, Profa. Dra. Sílvia R. C. J. Santos, Célia Etsuco K. Onosako, Dra. Mirian MagaIhães Pardi, Roseli O. Araújo. 
Pêgo-Fernandes P M, Jatene F B, Coelho F F, Gentil A F, Kawasnicka K L, Stolf N A G, Oliveira S A - Evolução hemodinâmica da revascularização do miocárdio com dois métodos de proteção miocárdica. Rev Bras Cir Cardiovasc 2000; 15(3): 212-8.

RBCCV 44205-504

Pêgo-Fernandes P M, Jatene F B, Coelho F F, Gentil A F, Kawasnicka K L, Stolf N A G, Oliveira S A Hemodynamic evolution of myocardial revascularization through two methods of myocardial protection. Rev Bras Cir Cardiovasc 2000; 15(3): 212-8.

SUMMARY: Objective: The aim of this study is to evaluate immediate hemodynamics evolution in myocardial revascularization with intermittent aortic cross-clamping, using or not a preconditioning protocol.

Material and Methods: Thirty-five patients underwent myocardial revascularization, they were randomized in two groups: the control group (18) with intermittent aortic-clamping, and the preconditioning group (17) with two short aortic-clamping before the onset of conventional intermittent clamping. In both groups the patients' mean arterial pressure (mAP), pulmonary capillary wedge pressure (PCWP), cardiac index (Cl), left ventricular stroke work index (LVSWI) were monitored before the onset of the cardiopulmonary bypass (moment 1 ) and the sternal suture (moment 2), and, postoperatively at six/twelve/eighteen/twenty-four hours (moments 3, 4, 5 and 6, respectively). Also the patients' left ventricular ejection fraction (LVEF) was controlled through echocardiogram, in moments 1 and 2.

Results: None statistical differences was seen between groups, however occurred a growth in $\mathrm{Cl}$ and LVEF after revascularization. All patients presented good clinical evolution.

Conclusion: The hemodynamic evolution was similar between the groups.

DESCRIPTORS: Myocardial revascularization. Myocardial revascularization, methods. Hemodynamics, physiology. Extracorporeal circulation. Aortic clamping. Myocardial protection. Myocardial revascularization, comparative study. Myocardial revascularization, hemodynamics.

\section{REFERÊNCIAS BIBLIOGRÁFICAS}

1 Reimer K A, Murry C E, Yamasawa I, Hill M L, Jennings $\mathrm{R} B$ - Four brief periods of myocardial ischemia cause no cumulative ATP loss or necrosis. Am J Physiol 1986; 251(6 Pt 2): H1306-15.

2 Henrichs K J, Matsuoka H, Schaper J - Influence of repetitive coronary occlusions on myocardial adenine nucleosides, high energy phosphates and ultrastructure. Basic Res Cardiol 1987; 82: 557-65.

3 Murry C E, Jennings R B, Reimer K A - Preconditioning with ischemia: a delay of lethal cell injury in ischemic myocardium. Circulation 1986; 74: 1124-36.

4 Cremer J, Steinhoff $G$, Karck $M$ et al. - Ischemic preconditioning prior to myocardial protection with cold blood cardioplegia in coronary surgery. Eur $J$ Cardiothorac Surg 1997; 12: 753-8.

$5 \quad$ Niroomand F \& Kubler W - Hibernating, stunning and ischemic preconditioning of the myocardium: therapeutic implications. Clin Investig 1994; 72: 731-6.

6 Illes R W \& Swoyer K D - Prospective, randomized clinical study of ischemic preconditioning as an adjunct to intermittent cold blood cardioplegia. Ann Thorac Surg 1998; 65: 748-53.

7 Chagas A C P, Galvão T F G, Ferreiro C R, Luz P L Pré-condicionamento isquêmico: um mecanismo protetor eficaz do coração em risco de necrose. Rev Soc Cardiol Estado de São Paulo 1998; 8: 314-28.

8 Bouchart F, Bessou J P, Tabley A et al. - How to protect hypertrophied myocardium? A prospective clinical trial of three preservation techniques. Int $J$ Artif Organs 1997; 20: 440-6.

9 Tomai F, Crea F, Gaspardone A et al. - Ischemic preconditioning during coronary angioplasty is prevented by glibenclamide, a selective ATP. sensitive K+ channel blocker. Circulation 1994; 90: 700-5.

10 Jenkins D P, Pugsley W B, Alkhulaifi A M, Kemp M, Hooper J, Yellon D M - Ischaemic preconditioning reduces troponin $\mathrm{T}$ release in patients undergoing coronary artery bypass surgery. Heart 1997; 77: 314-8.

11 Vegh A, Komori S, Szekeres L, Parratt J R Antiarrhythmic effects of preconditioning in anaesthetised dogs and rats. Cardiovasc Res 1992; 26: 487-95.

12 Gerola L R, Oliveira S A, Moreira L F et al. - Blood cardioplegia with warm reperfusion versus intermittent aortic crossclamping in myocardial revascularization: randomized controlled trial. J Thorac Cardiovasc Surg 1993; 106: 491-6.

13 Levitsky S, Wright R N, Rao K S et al. - Does intermittent coronary perfusion offer greater myocardial protection than continuous aortic cross-clamping? Surgery 1977; 82: $51-9$.

14 Flameng W, Van der Vusse G J, De Meyere R et al. - Intermittent aortic cross-clamping versus St. Thomas' Hospital cardioplegia in extensive aortacoronary bypass grafting: a randomized clinical study. J Thorac Cardiovasc Surg 1984; 88: 164-73. 
Pêgo-Fernandes P M, Jatene F B, Coelho F F, Gentil A F, Kawasnicka K L, Stolf N A G, Oliveira S A - Evolução hemodinâmica da revascularização do miocárdio com dois métodos de proteção miocárdica. Rev Bras Cir Cardiovasc 2000; 15(3): 212-8.

15 Malkowski M J, Kramer C M, Parvizi S T et al. - Transient ischemia does not limit subsequent ischemic regional dysfunction in humans: a transesophageal echocardiographic study during minimally invasive coronary artery bypass surgery. J Am Coll Cardiol 1998; 31: 1035-9.

16 Tomoda $\mathrm{H} \&$ Aoki $\mathrm{N}$ - Comparison of protective effects of preinfarction angina pectoris in acute myocardial infarction treated by thrombolysis versus by primary coronary angioplasty with stenting. Am J Cardiol 1999; 84: $621-5$.
17 Hirai T, Fujita M, Yoshida N, Yamanishi K, Inoko M, Miwa K - Importance of ischemic preconditioning and collateral circulation for left ventricular functional recovery in patients with successful intracoronary thrombolysis for acute myocardial infarction. Am Heart J 1993; 126: 827-31.

18 Antelmi I, Kalil R, Lopes $\mathrm{N} \mathrm{H}$ et al. - Evaluation of ischemic preconditioning on collateral circulation, ventricular function and clinical outcome in acute myocardial infarction. Arq Bras Cardiol 1996; 66: 11-4. 